\title{
GP involvement after a cancer diagnosis; patients' call to improve decision support
}

\author{
Eveline A Noteboom ${ }^{1 *}$, letje AA Perfors ${ }^{1}$, Anne M May', Mariken E Stegmann², \\ Saskia FA Duijts ${ }^{3,4}$, Ella A Visserman ${ }^{5}$, Vivian Engelen ${ }^{5}$, Carol Richel ${ }^{6}$, \\ Elsken van der Wall ${ }^{7}$, Niek de Wit ${ }^{1}$, Charles W Helsper ${ }^{1}$
}

${ }^{1}$ Julius Center for Health Sciences and Primary Care, University Medical Centre Utrecht, Utrecht, the Netherlands; ${ }^{2}$ Department of General Practice and Elderly Care Medicine, University Medical Center Groningen, Groningen, the Netherlands; ${ }^{3}$ Netherlands Comprehensive Cancer Organisation, Utrecht, the Netherlands; ${ }^{4}$ Public and Occupational Health, Amsterdam UMC, Amsterdam, the Netherlands; ${ }^{5}$ Dutch Federation of Cancer Patient Organisations, Utrecht, the Netherlands; ${ }^{6}$ Dutch Breast Cancer Association, Utrecht, the Netherlands; ${ }^{7}$ Department of Medical Oncology, University Medical Centre Utrecht, Utrecht, the Netherlands

*For correspondence: gripstudie@umcutrecht.nl

Competing interest: The authors declare that no competing interests exist.

Received: 24 April 2020

Accepted: 13 May 2020

Published: 09 December 2020

(C)This article is Open Access: CC BY license (https://creativecommons.org/licenses/by/4.0/)

Author Keywords: neoplasms, general practice, decision making, shared

Copyright (C) 2020, The Authors; DOI:10.3399/

bjgpopen20X101124

\section{Abstract}

Background: Shared decision making (SDM) is considered important to realise personalised cancer care. Increased GP involvement after a diagnosis is advocated to improve SDM.

Aim: To explore whether patients with cancer are in need of GP involvement in cancer care in general and in SDM, and whether GP involvement occurs.

Design \& setting: An online national survey was distributed by the Dutch Federation of Cancer Patient Organisations (NFK) in May 2019.

Method: The survey was sent to (former) patients with cancer. Topics included GP involvement in cancer care in general and in SDM. Descriptive statistics and quotes were used.

Results: Among 4763 (former) patients with cancer, 59\% ( $n=2804$ ) expressed a need for GP involvement in cancer care. Of these patients, 79\% $(n=2193)$ experienced GP involvement. Regarding GP involvement in SDM, $82 \%$ of patients $(n=3724)$ expressed that the GP should 'listen to patients' worries and considerations', $69 \%(n=3130)$ to 'check patients' understanding of information', 66\% $(n=3006)$ to 'discuss patients' priorities in life and the consequences of treatment options for these priorities', and $67 \%(n=3045)$ to 'create awareness of the patient's role in the decision making'. This happened in $47 \%, 17 \%, 15 \%$ and $10 \%$ of these patients, respectively.

Conclusion: The majority of (former) patients with cancer expressed a need for active GP involvement in cancer care. GP support in the fundamental SDM steps is presently insufficient. Therefore, GPs should be made aware of these needs and enabled to support their patients with cancer in SDM.

\section{How this fits in}

Little is known about patients' needs for GP involvement in cancer care and in shared decision making (SDM), and to what extent GP involvement occurs. This study showed that the majority of (former) patients with cancer had a need for GP involvement in cancer care and in SDM. However, GP involvement in SDM was infrequently experienced. Therefore, GPs should be made aware of these needs and enabled to support their patients to make personalised cancer treatment decisions. 


\section{Introduction}

Cancer treatment decisions have become more complex, owing to the increasing number of treatment options. This enables a more personalised approach. ${ }^{1}$ Incorporating personal preferences in treatment decisions requires SDM. SDM aims to establish a treatment decision that optimally matches a patient's personal preferences and expectations. ${ }^{2}$ An effective SDM process consists of four steps: 1) awareness of choice; 2) explanation of treatment options; 3) time for deliberation; and 4) making an informed decision. $^{2}$

Unfortunately, in the present hospital-oriented cancer care pathway, essential steps for successful SDM are usually insufficiently supported. First, patients with cancer are often unaware of their important role in choosing the 'best-fitting' treatment. ${ }^{3}$ Second, medical information, including treatment options, is often not understood by patients with cancer. ${ }^{4}$ Third, time for deliberation is often limited, since the short in-hospital pathway between diagnosis and treatment choice generally does not facilitate reflection. This leaves little room to consider treatment options in the light of patients' personal preferences and expectations. ${ }^{3,5,6}$

GPs usually have longstanding relationships with their patients. Consequently, for many, the GP is the 'trusted healthcare professional', with longitudinal knowledge of their patients' medical and personal history. ${ }^{1,7}$ Hence, the GP is considered to be in the ideal position to guide the patient through the different steps of the SDM process. ${ }^{1,6}$ Patients with cancer and GPs support this extended role for the GP in cancer treatment decision making; for example, through determining patients' preferences, discussing treatment options, and explaining medical information. ${ }^{8-10}$

Positive effects of increased GP involvement after a cancer diagnosis have been described previously. Wallner et al showed that a patient's experience of GP engagement, that is, how informed the patient felt the GP was about the diagnosis, was associated with higher satisfaction of treatment decisions in cancer. ${ }^{11}$ Wieldraaijer et al showed that a consultation with the GP between diagnosis and start of treatment is beneficial for patients' feelings of comfort and satisfaction. ${ }^{12}$ It has been demonstrated earlier by the authors of the present study that a cancer-related GP consultation before treatment decision may improve the SDM process of palliatively treated patients with cancer, according to patients, GPs, and treating physicians. ${ }^{13}$

Despite this broadly shared call for more GP involvement in the process of making cancer treatment decisions, little is known about patients' perspectives. Therefore, the study aimed to explore patients' needs for GP involvement after a cancer diagnosis in general and in SDM, and whether this GP involvement occurred.

\section{Method}

\section{Design}

An online national survey was developed and distributed among (former) patients with cancer in the Netherlands in May 2019 by the NFK.

\section{Study population}

NFK is an umbrella organisation of 19 cancer patient organisations. These organisations together represent approximately 35000 (former) patients with cancer. The survey was distributed in several ways. First, the survey was dispersed to the affiliated cancer patient organisations, which represent adult patients with a large variety in diagnoses. These organisations were asked to distribute the survey among their members. This could either be directly to all members or indirectly through their newsletter. Second, a web link to the survey was distributed through social media accounts of NFK (Facebook, Linkedln, Twitter, and Instagram), via their website, and via other relevant partner organisations (such as The Dutch Cancer Society and the website kanker.nl). Finally, a panel of (former) patients with cancer, who were not members of one of the cancer patient organisations, were sent invitations to participate in the survey. These patients registered voluntarily to receive invitations for NFK surveys and were not selected for this specific survey.

\section{Online survey}

The online survey was developed by NFK, in cooperation with experts in the fields of cancer, primary care, and SDM, including patients, clinicians, researchers, and policymakers. The survey consisted of 
the following two parts: one part focused on the role of the GP; and the other on the role of the specialised oncology nurse. This study only used data from the GP-related questions.

The survey started with a selection question, only participants who responded yes to the question, 'Do, or did, you have cancer?' were able to proceed with filling in the questionnaire. Then, eight general questions about patient and disease characteristics followed. Hereafter, 10 questions addressing the patient's personal needs for GP involvement in cancer care were posed. These questions covered the following topics: 1) the need for GP involvement in cancer care at any time after diagnosis; 2 ) whether this GP involvement occurred; 3 ) the need to have SDM topics addressed in a GP consultation; and 4) whether these topics were actually addressed. Finally, the survey assessed 5) the initiator of involvement of the GP in cancer care and 6) satisfaction with GP involvement in cancer care (see Supplementary Appendix S1 for the survey).

GP involvement in cancer care was defined as: 'Any type of long or short contact with the GP about the diagnosis, treatment and/ or its consequences. This could either be via telephone, an appointment at the GP's office, or a home-visit.' The SDM topics included that the GP should: 1) 'Listen to my worries and considerations about the diagnosis, treatment and its consequences'; 2) 'Check if I understand the information about my diagnosis, treatment and its consequences'; 3) 'Discuss what I think is important in my life and the consequences of treatment options for these priorities'; and 4) 'Explain to me the importance of my own opinion when making a treatment decision'.

The format of the questions was either closed (numeric, multiple choice) or open-ended. Needs and the occurrence of GP involvement were assessed with multiple-choice questions and open-ended questions for clarification. Satisfaction with GP involvement in cancer care was scored on a 10-point number rating scale ranging from 1 (very unsatisfied) to 10 (very satisfied). The estimated time to complete the questionnaire was approximately 5-10 minutes. The data were collected with the online tool SurveyMonkey. Responders participated anonymously in the survey. The survey was open for response for 2 weeks. Responders could choose to answer only part of the questions. Only if the general questions and the question, 'Did you have a need for contact with your GP about your cancer diagnosis, the treatment and/or its consequences?' were answered with 'yes', 'no' or 'don't know/NA', the survey was used in the analysis.
Table 1 Baseline characteristics of responders

Total, $\mathbf{N}=\mathbf{4 7 6 3}$

\begin{tabular}{lcc}
\hline & $n$ & $\%$ \\
\hline Female & 2686 & 56 \\
\hline $\begin{array}{l}\text { Age, years, mean }( \pm \\
\text { SD) }\end{array}$ & 62 & $( \pm 12)$ \\
\hline Education $^{\mathrm{a}}$ & & \\
\hline High & 2276 & 48 \\
\hline Middle & 1908 & 40 \\
\hline Low & 464 & 10 \\
\hline Other & 61 & 1 \\
\hline Missing & 54 & 1
\end{tabular}

Diagnosis

\begin{tabular}{|c|c|c|}
\hline Breast cancer & 1231 & 26 \\
\hline $\begin{array}{l}\text { Haematological } \\
\text { cancers }\end{array}$ & 874 & 18 \\
\hline Colorectal cancer & 787 & 16 \\
\hline Prostate cancer & 569 & 12 \\
\hline Bladder cancer & 270 & 6 \\
\hline Gynaecologic cancer & 179 & 4 \\
\hline Lung cancer & 153 & 3 \\
\hline Melanoma & 125 & 3 \\
\hline Esophageal cancer & 105 & 2 \\
\hline Other & 470 & 10 \\
\hline $\begin{array}{l}\text { Years since last } \\
\text { received cancer } \\
\text { treatment, median } \\
(\mathrm{OQR})\end{array}$ & 2 & $1-6$ \\
\hline
\end{tabular}

Patient reported

cancer stage

\begin{tabular}{lcc}
\hline Cured & 2166 & 46 \\
\hline Will probably be cured & 901 & 19 \\
\hline $\begin{array}{l}\text { Will probably not be } \\
\text { cured }\end{array}$ & 1256 & 26 \\
\hline Don't know/NA & 440 & 9 \\
\hline
\end{tabular}

${ }^{a}$ Education is categorised as high (university or higher professional education), middle (secondary education), and low (primary education or no education). IQR = interquartile range. $\mathrm{NA}=$ not applicable. $\mathrm{SD}=$ standard deviation. 


\section{Analysis}

Descriptive analyses of the closed questions were performed for the total population and for subgroups of the following characteristics: sex, age, education, type of cancer, cancer stage, and time since last treatment. Statistical testing was not performed, since with the current number of patients, small often not (clinically) relevant differences would already be statistically significant. Categorical variables are presented as numbers and percentages. Continuous variables are presented, depending on whether or not normally distributed, with means and standard deviations (SD) or medians and interquartile ranges (IQR). All analyses were performed with IBM SPSS Statistics (version 25). Relevant quotes from the open questions were used to illustrate the results.

\section{Results}

\section{Patient characteristics}

The survey was completed by 4763 (former) patients with cancer. The mean age of responders was 62 years (SD \pm 12 ), $56 \%$ were female, and $48 \%$ of the responders had a high education level (Table 1). The majority of the responders were diagnosed with either breast cancer (26\%), haematological cancers $(18 \%)$, or colorectal cancer $(16 \%)$. The median time since the last received cancer treatment was 2 years (IQR 1-6) and $46 \%$ reported to be cured.

\section{GP involvement in general}

Of all responders, 59\% ( $n=2804)$ expressed a need for GP involvement in cancer care any time after diagnosis (Table 2). GP involvement in cancer care was experienced by $79 \%(n=2193)$ of these responders. A relatively high need for GP involvement was reported by females (female: 64\%; male: $52 \%)$. GP involvement occurred more often in males (82\%) than in females (77\%). A relatively high need for GP involvement was reported by patients with lung, oesophageal, and gynaecologic cancer (68\%$69 \%)$, versus other cancers (47\%-64\%). A relatively small proportion of (former) patients with breast and gynaecologic cancer experienced GP involvement (74\%-76\%), compared with other cancers (78\%-88\%). Responders who indicated 'will probably not be cured' reported relatively high need of GP involvement (66\%) compared with those who indicated to be 'cured' (55\%). The latter group reported GP involvement less often (75\% versus 85\%). Quotes in Supplementary Box S1 illustrate the need for, and lack of, experiences with GP involvement in cancer care.

\section{GP involvement in SDM}

Table 3 shows the needs to have SDM topics addressed in a GP consultation and whether these topics were actually addressed. Eighty-two per cent $(n=3724)$ of the responders expressed that their GP should listen to their worries and considerations about the diagnosis, treatment, and its consequences. This actually happened in $47 \%(n=1744)$ of these cases. The majority of the responders expressed that the GP should: 'check understanding of information' 69\% ( $n=3130)$; 'discuss patients' priorities in life and the consequences of treatment options for these priorities' $66 \%(n=3006)$; and 'explain importance of patients' opinions in decisions' $67 \%(n=3045)$. These topics were addressed in respectively $17 \%(n=542), 15 \%(n=461)$, and $10 \%(n=294)$ of these cases.

In all subgroups, the need for GP involvement in the SDM process was high. However, this GP involvement in SDM was infrequently experienced by responders, especially by responders aged $\geq 65$ years, those with low education, those with breast, bladder, gynaecologic, haematological cancers, or colon cancer, and by the 'cured' group of responders. Quotes that illustrate the need for GP involvement in SDM are presented in Supplementary Box S1.

\section{Initiator and satisfaction}

Among those who reported that their GP was involved in cancer care, this was initiated by the patient in $52 \%(n=1650)$, by the GP in $31 \%$ ( $n=987)$, by family and friends in $4 \%(n=116)$, and unknown in $13 \%(n=421)$ (data not shown). In case of GP involvement, satisfaction with GP involvement in cancer care was evaluated with a mean score of $7.4( \pm 2.4)$. This involvement was rated higher if the GP was the initiator $(8.0 \pm 2.0)$, instead of the patient $(7.0 \pm 2.4)$. This is illustrated by the final quote in Supplementary Box S1. 
Table 2 Need for GP involvement in cancer care and whether GP involvement occurred. Presented for total and stratified per subgroup

\begin{tabular}{|c|c|c|c|c|c|}
\hline \multicolumn{6}{|c|}{ Need for GP involvement in cancer care any time after diagnosis } \\
\hline & & \multicolumn{2}{|c|}{ Need (yes) } & \multicolumn{2}{|c|}{ Contact occurred? (yes) } \\
\hline & \multirow{2}{*}{$\begin{array}{c}\text { Total } \\
n \\
\end{array}$} & \multicolumn{2}{|c|}{ Of total } & \multicolumn{2}{|c|}{ Of need } \\
\hline & & $n$ & $\%$ & $n$ & $\%$ \\
\hline All responders & 4763 & 2804 & 59 & 2193 & 79 \\
\hline \multicolumn{6}{|l|}{ Sex } \\
\hline Male & 2077 & 1073 & 52 & 873 & 82 \\
\hline Female & 2686 & 1731 & 64 & 1320 & 77 \\
\hline \multicolumn{6}{|l|}{ Age } \\
\hline Aged $<65$ years & 2537 & 1577 & 62 & 1245 & 80 \\
\hline Aged $\geq 65$ years & 2226 & 1227 & 55 & 948 & 78 \\
\hline \multicolumn{6}{|l|}{ Education } \\
\hline Low education ${ }^{b}$ & 464 & 254 & 55 & 188 & 75 \\
\hline Middle education ${ }^{b}$ & 1908 & 1134 & 59 & 849 & 76 \\
\hline High education ${ }^{\mathrm{b}}$ & 2276 & 1351 & 59 & 1105 & 82 \\
\hline \multicolumn{6}{|l|}{ Diagnosis } \\
\hline Haematological cancers & 874 & 478 & 55 & 380 & 80 \\
\hline Colorectal cancer & 787 & 402 & 51 & 307 & 78 \\
\hline Bladder cancer & 270 & 128 & 47 & 105 & 83 \\
\hline Gynaecologic cancer & 179 & 121 & 68 & 91 & 76 \\
\hline Melanoma cancer & 125 & 75 & 60 & 64 & 85 \\
\hline Breast cancer & 1231 & 791 & 64 & 582 & 74 \\
\hline Prostate cancer & 569 & 323 & 57 & 276 & 86 \\
\hline Lung cancer & 153 & 105 & 69 & 83 & 81 \\
\hline Oesophageal cancer & 105 & 72 & 69 & 63 & 88 \\
\hline
\end{tabular}

Years since last received cancer treatment

\begin{tabular}{lccccc}
\hline Last treatment $\leq 2$ years ago & 2404 & 1462 & 61 & 1215 & 84 \\
\hline Last treatment $\geq 3$ years ago & 2359 & 1342 & 57 & 978 & 74 \\
\hline Patient reported cancer stage & & & & & 75 \\
\hline Cured & 2166 & 1180 & 55 & 875 & 78 \\
\hline Will probably be cured & 901 & 535 & 59 & 413 & 85 \\
\hline Will probably not be cured & 1256 & 825 & 66 & 699 & 75 \\
\hline
\end{tabular}

aPercentage 'Contact occurred? (yes)' is calculated for those who responded to have a need for GP involvement and filled in the question 'Contact occurred?'. Denominators vary for this question and are slightly lower than the total number of patients who indicated to have a need for GP involvement, due to missing data (i.e. not al respondents filled in the follow-up question 'Contact occurred'). Further information is available from the authors on request ${ }^{b} E d u c a t i o n$ is categorised as high (university or higher professional education), middle (secondary education), and low (primary education or no education). 


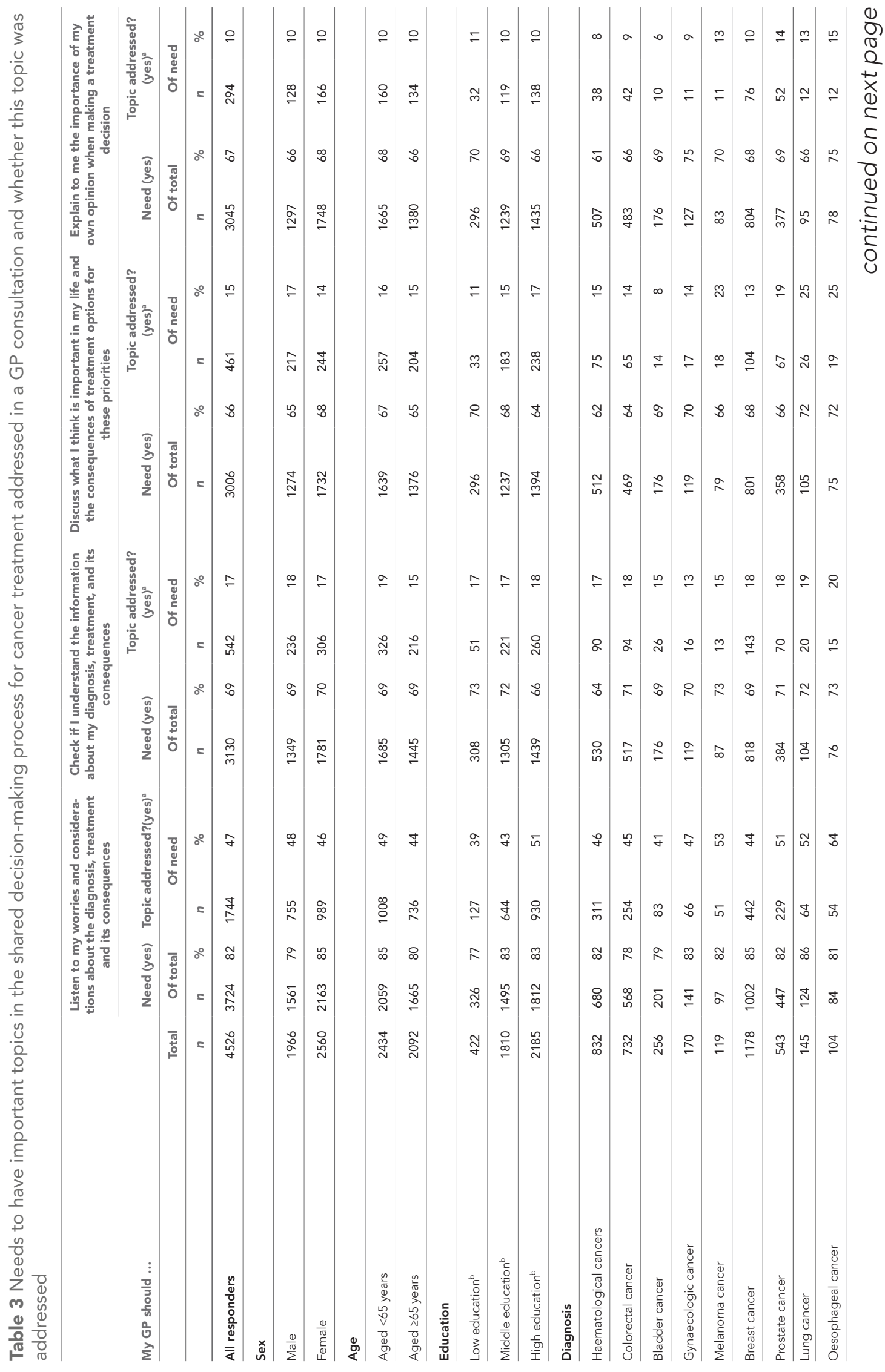




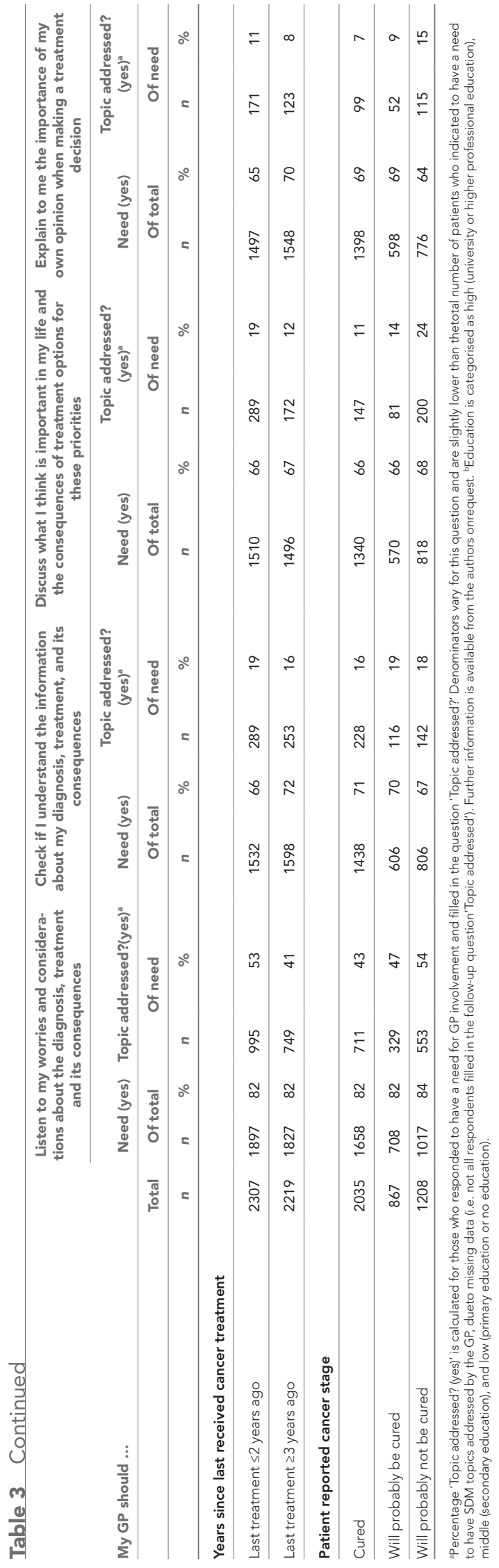




\section{Discussion}

\section{Summary}

In the present study, the needs of (former) patients with cancer were evaluated for GP involvement in cancer care. More than half of the responders reported that they wanted the GP to be involved in cancer care after the diagnosis. GP involvement in cancer care was experienced in over threequarters of these cases. As for GP involvement in SDM for cancer treatment, the balance is different. Although more than $80 \%$ expressed a need for the GP to listen to worries and considerations, this support was lacking in just over half of these cases. Also, more than two-thirds of responding patients with cancer indicated a need to have elemental SDM topics addressed in a GP consultation, such as explaining information, checking understanding, and discussing priorities. This SDM support was only experienced in a small minority of cases. Finally, the initiator of GP involvement was mostly the patient, whereas satisfaction with GP involvement in cancer care was higher if the GP was the initiator.

\section{Strengths and limitations}

This study has several limitations. First of all, recall bias may have occurred, since the median interval between last received treatment and participation was 2 years. Among those treated longer ago $(\geq 3$ years) the reported needs were similar to those treated $\leq 2$ years ago. However, those treated $\geq 3$ years ago reported GP involvement less often. This could be the result of an underestimation of the actual GP involvement, owing to incorrect recall. Second, the network used to recruit patients with cancer may have addressed a selective population. The survey was distributed among a group of (former) patients with cancer who are in some way affiliated to a cancer patient organisation. Consequently, the responders may have been relatively committed, active, and critical, thus may have different needs than the average patient with cancer and have a stronger drive to meet those needs. Within this population, selective response may have occurred, as those being very satisfied or unsatisfied with GP involvement may be more inclined to participate in a survey about corresponding needs. Selective participation is supported by the relatively high percentage of patients with a high education (48\%). However, the percentage of females (56\%) and the average age (62 years) in the sample is comparable with the Dutch population of patients with cancer. ${ }^{14}$

The main strength of this study is the high number of (former) patients with cancer who responded to this survey. The large population and the variety of cancer types support generalisability and enabled subgroup explorations.

\section{Comparison with existing literature}

To the authors' knowledge, this is the first study among (former) patients with cancer that combines an exploration of the needs for GP involvement in cancer care and specifically in SDM, and to what extent GP involvement occurred. The findings are in line with the few studies that have addressed adjacent topics. It confirms the need for a supportive role of the GP as previously demonstrated. ${ }^{10,15}$ It also confirms the conclusion of Halkett et al, who reported that patients with cancer see a role for the GP in SDM support after a cancer diagnosis. ${ }^{10}$ Lang et al reported that $34.5 \%$ of the patients with cancer discussed diagnostic and therapy-related decisions with the GP. ${ }^{9}$ Also, Klabunde et al showed that $64.2 \%$ of the GPs reported to explore patients' preferences for treatment. ${ }^{8}$ Both percentages are higher compared with the $15 \%$ of the (former) patients with cancer who reported SDM involvement by the GP in the present study. This might be owing to a different study population or because of differences in perception between GPs and patients of what is actually addressed during the consultation. Additionally, the results imply that GPs generally provide supportive care, mostly including the discussion of worries and considerations, but the discussion of the cancer treatment decision itself is often lacking. This might be caused by GPs' unawareness of patients' needs for SDM support, or by reluctance among GPs because of perceived lack of expertise. ${ }^{13,16}$

Furthermore, the results show that satisfaction with GP involvement is scored higher if the GP is the initiator of contact. This is supported by findings in a qualitative study by Brandenbarg et al among curatively treated patients with colorectal cancer who expressed dislike when the GP did not initiate contact after treatment. ${ }^{15}$ Also, cancer patients' preferences for initiation of contact by the GP is expressed for other conversations, such as for advanced care planning. ${ }^{17}$ In addition, previous studies show that patients with cancer are more satisfied if the GP is informed about the diagnosis ${ }^{11}$ and if 
there is a contact moment with the GP (a 'time-out consultation') before the start of treatment. ${ }^{12}$ The findings also support and explain the potential positive effect on SDM of actively involving the GP between diagnosis and therapy choice, which was recently reported for palliatively treated patients with cancer. ${ }^{13}$

\section{Implications for practice}

Treating physicians and GPs should actively explore patients' needs for GP involvement after a cancer diagnosis, particularly for SDM. GPs should be aware that patients wish to have cancer treatment decision-related topics addressed by the GP. GP support could be enabled to support SDM in the hospital, for instance, by actively offering a 'time-out consultation' with the GP with SDM tools. ${ }^{12,13,18,19}$ In addition, cancer patient organisations could support GP involvement by empowering patients to discuss preferred topics with their GP.

In conclusion, even though patients experience GP support after a cancer diagnosis, their needs for support in the SDM process often remain unanswered. GPs can do better in checking understanding of information, discussing patients' priorities and preferences, and explaining the importance of patients' own opinions in decision making. Since GPs seem adequately equipped to provide the desired SDM support, GPs and hospitals should join forces to make sure that GPs can and will support their patients when faced with important medical decisions.

\section{Funding}

None.

\section{Ethical approval}

As this study did not involve an intervention, the Medical Research Human Subject Acts does not apply. All procedures performed were in accordance with the 1964 Helsinki declaration, its amendments, and comparable ethical standards.

\section{Provenance}

Freely submitted; externally peer reviewed.

Acknowledgements

The authors are thankful to all participants of this study.

\section{References}

1. Nederlands Huisartsen Genootschap (NHG). NHG-Standpunt: Oncological care in general practice. Utrecht: NHG; 2014.

2. Elwyn G, Frosch D, Thomson R, et al. Shared decision making: a model for clinical practice. J Gen Intern Med 2012; 27(10): 1361-1367. DOI: https://doi.org/10.1007/s11606-012-2077-6

3. Brom L, De Snoo-Trimp JC, Onwuteaka-Philipsen BD, et al. Challenges in shared decision making in advanced cancer care: a qualitative longitudinal observational and interview study. Health Expect 2017; 20(1): 69-84. DOI: https://doi.org/10.1111/hex.12434

4. Weeks JC, Catalano PJ, Cronin A, et al. Patients' expectations about effects of chemotherapy for advanced cancer. N Engl J Med 2012; 367(17): 1616-1625. DOI: https://doi.org/10.1056/NEJMoa1204410

5. KNMG. Not all that's possible, has to be done. Utrecht: KNMG; 2015.

6. de Wit NJ. A "time out consultation" in primary care for elderly patients with cancer: better treatment decisions by structural involvement of the general practitioner. Eur J Cancer Care 2017; 26(3): e12711. DOI: https://doi.org/10. 1111/ecc.12711

7. Rubin G, Berendsen A, Crawford SM, et al. The expanding role of primary care in cancer control. Lancet Oncol 2015; 16(12): 1231-1272. DOI: https://doi.org/10.1016/S1470-2045(15)00205-3

8. Klabunde $\mathrm{CN}$, Ambs A, Keating NL, et al. The role of primary care physicians in cancer care. J Gen Intern Med 2009; 24(9): 1029-1036. DOI: https://doi.org/10.1007/s11606-009-1058-x

9. Lang V, Walter S, Fessler J, et al. The role of the general practitioner in cancer care: a survey of the patients' perspective. J Cancer Res Clin Oncol 2017; 143(5): 895-904. DOI: https://doi.org/10.1007/s00432-017-2343-4

10. Halkett GKB, Jiwa M, Lobb EA. Patients' perspectives on the role of their general practitioner after receiving an advanced cancer diagnosis. Eur J Cancer Care 2015; 24(5): 662-672. DOI: https://doi.org/10.1111/ecc.12224

11. Wallner LP, Abrahamse $P$, Uppal JK, et al. Involvement of primary care physicians in the decision making and care of patients with breast cancer. J Clin Oncol 2016; 34(33): 3969-3975. DOI: https://doi.org/10.1200/JCO.2016.67. 8896 
12. Wieldraaijer T, Meij M, Zwaard S, et al. Introducing a time out consultation with the general practitioner between diagnosis and start of colorectal cancer treatment: patient-reported outcomes. Eur J Cancer Care 2019; 28(6): e13141. DOI: https://doi.org/10.1111/ecc.13141

13. Noteboom EA, de Wit NJ, van Asseldonk IJEM, et al. Off to a good start after a cancer diagnosis: implementation of a time out consultation in primary care before cancer treatment decision. J Cancer Surviv 2020; 14(1): 9-13. DOI: https://doi.org/10.1007/s11764-019-00814-5

14. 14. 14. 14. Nederlands IK (Netherlands Comprehensive Cancer Organisation). Cancer rates. 2018; https:// www.cijfersoverkanker.nl/ (accessed 3 Dec 2020).

15. Brandenbarg D, Roorda C, Stadlander M, et al. Patients' views on general practitioners' role during treatment and follow-up of colorectal cancer: a qualitative study. Fam Pract 2017; 34(2): 234-238. DOI: https://doi.org/10.1093/ fampra/cmw124

16. Anvik T, Holtedahl KA, Mikalsen $\mathrm{H}$. "When patients have cancer, they stop seeing me" — the role of the general practitioner in early follow-up of patients with cancer - a qualitative study. BMC Fam Pract 2006; 7(1): 19. DOI: https://doi.org/10.1186/1471-2296-7-19

17. Stegmann ME, Geerse OP, Tange D, et al. Experiences and needs of patients with incurable cancer regarding advance care planning: results from a national cross-sectional survey. Support Care Cancer 2020; 28(9): 4211-4217. DOI: https://doi.org/10.1007/s00520-019-05285-z

18. Stegmann ME, Festen S, Brandenbarg D, et al. Using the outcome prioritization tool (OPT) to assess the preferences of older patients in clinical decision-making: a review. Maturitas 2019; 128: 49-52. DOI: https://doi. org/10.1016/j.maturitas.2019.07.022

19. Perfors IAA, Helsper CW, Noteboom EA, et al. Randomised controlled trial protocol (grip study): examining the effect of involvement of a general practitioner and home care oncology nurse after a cancer diagnosis on patient reported outcomes and healthcare utilization. BMC Cancer 2018; 18(1): 132. DOI: https://doi.org/10.1186/s12885018-4005-6 